\title{
WIRELESS AUDIO TRANSMISSION AND SWITCHING SYSTEM FOR
}

\section{PUBLIC ADDRESS}

\author{
Shubham Kumbhalkar ${ }^{1}$, Aditya Malviya ${ }^{2}$, Sagar Waghale ${ }^{3}$, Gaurang Joshi ${ }^{4}$ \\ ${ }^{1}$ St. Vincent Pallotti College of Engineering and Technology \\ ${ }^{2}$ St. Vincent Pallotti College of Engineering and Technology \\ ${ }^{3}$ St. Vincent Pallotti College of Engineering and Technology \\ ${ }^{4}$ St. Vincent Pallotti College of Engineering and Technology
}

\begin{abstract}
The world is being digitalized so is the advancement in field of communication. Wireless communication plays a vital role in this digital world. Point to point communication (single transmitter single receiver) has led to Multi-point communication (single transmitter multiple receiver) which is the need of this modern world. This paper discuss details related to transmission of audio signals through wireless medium using Radio Frequency. The paper is aimed at developing "Wireless Audio Transmission and Switching System for Public Address". Most of the devices available now which are present in wireless system works on Bluetooth and Wi-Fi. This restricts their range and create issues regarding bandwidth. The device discussed here will be able to send the data over a range of 60-100 meters (LOS).The microcontroller is used for signal processing, configuration and switching of receiver.This product is cost effective and the price can be reduced to a more extent in case of mass production.
\end{abstract}

Keywords: Wireless Communication, Switching, Radio Frequency, Bandwidth, Wireless Audio.

\section{INTRODUCTION}

Communication is the basic need of all organisations whether it is Hospitals, Educational institutes or corporate companies. Wireless communication plays a vital role in this digital world. Point to point communication (single transmitter single receiver) has led to Multi-point communication (single transmitter multiple receiver) which is the need of this modern world. Multi-point communication controls multiple receivers with a single transmitter such that common data can be send to all receivers or if the data is private it will be send to the concerned receivers only. The world is being digitalized so the need for modern communication i.e. Advancement in the field of communication is necessary. The paper discusses details related to transmission of audio signals through wireless medium using Radio Frequency and how multipoint communication has a edge over simple communication which was involving only a single transmitter resulting in many drawbacks. This "Wireless Audio Transmission and Switching System for Public Address" will be working simply on the basic concept of wireless transmission using radio frequency. This project is aimed at developing a "Wireless Audio Transmission and Switching System for Public Address". Nearly all the available devices which are present in wireless system works on Bluetooth and Wi-Fi. This restricts their range, directivity and issues regarding bandwidth. The project discussed here will be able to send the data over a range of 60-100 meters (line of sight). The microcontroller is used to configure the receiver and also for signal processing. The fact by which it differs from conventional system is its efficiency, cost-efficient, power, range, multipoint transmission and reliability

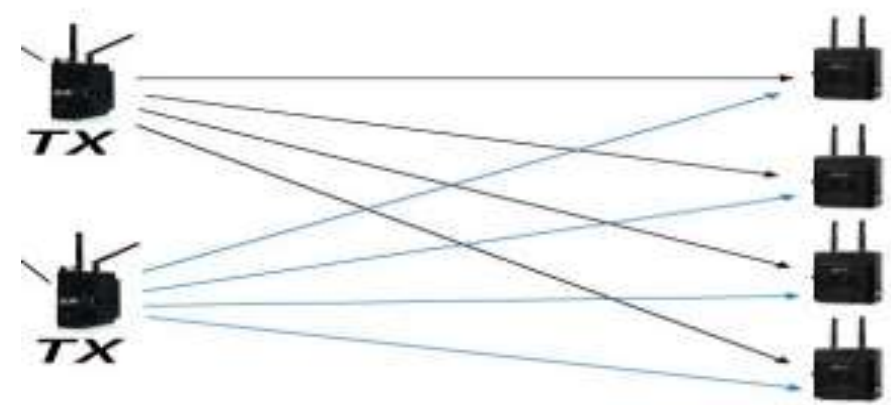

Fig 1. Basic concept for Tx and Rx

\section{DESIGN}

This product focuses on simple mechanism of wireless communication which involves transmitter, receiver, condenser mic and suspended speaker.

Sending the data after amplification through the antenna on the transmitter and get the data after amplification on the receiver side

The basic elements of our device are

1. Mic

2. Encoder

3. Amplifier

4. Antenna

5. Decoder

6. Microproccesor 


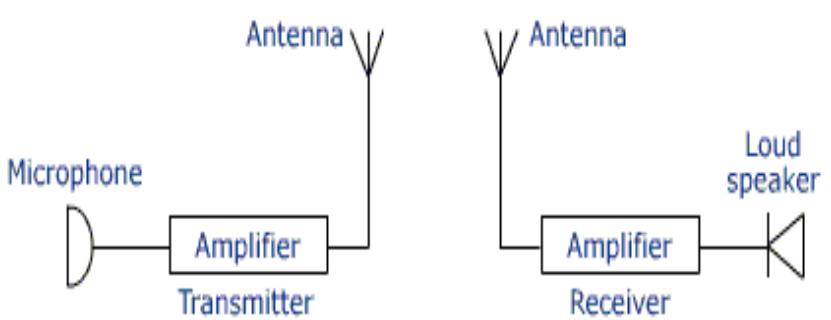

Fig 2. Transmitter and receiver
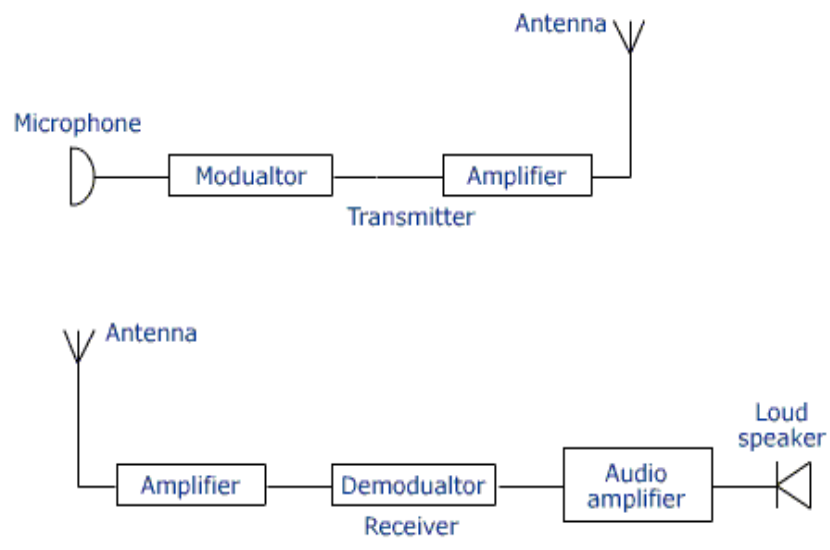

Fig 3. Encoding and Decoding concept

\section{WORKING}

Input in the form of audio signal is given to the condenser mic. Then the audio signal is converted to proportional electrical signal by mic.

The signal is then encoded in a appropriate form and then it is fed to further components.

The basic signal is so weak that it cannot be transmitted directly to a very long distance. Therefore then the electrical signal is the amplified and signal is then encoded in a appropriate form and then it is fed to the antenna. The antenna we are using is inbuilt in the transmitter module. Single point communication comprises of single transmitter and single receiver in which data is send to a particular receiver this has evolved to a concept of multipoint communication system in which multiple receivers are connected to a single transmitter as shown in figure. The data sent from the transmitter can be send individually to a particular receiver or all the receiver at a time. The transmitter can switch to receiver of his choice.

At the receiver side signal from the transmitter is then detected by the antenna on the receiver side and then fed to the decoder in the receiver section. Then the signal is decoded by the decoder. The output from the decoder is audio waveform but the signal is not so strong to drive the speaker so it is fed to audio amplifier the audio amplifier increase the power of the signal and fed to the suspended speakers. The signal which was sent is then will be accurately played on the speakers.

\section{FREQUENCY MODULATION}

In telecommunications and signal processing, the frequency modulation (FM) is the process in which the frequency of the career changes in accordance with the modulating signal. This is oppositeto amplitude modulation where the amplitude is varied keeping the frequency constant. But in FM we keep amplitude constant and vary the frequency.

In an analog frequency modulation, e.g. FM radio in which the broadcasting of an audio signal is represented by voice or music, here the instantaneous frequency deviation, the difference in the frequency of the carrier and its middle frequency, is thus proportional to the modulating signal.

Digital data is encoded and transmitted using FM by shifting the carrier's frequency among a predefined set of frequencies which represents digits - e.g. one frequency represents a binary 1 and a second represent binary 0 . This modulation technique is thus known as frequency-shift keying (FSK).

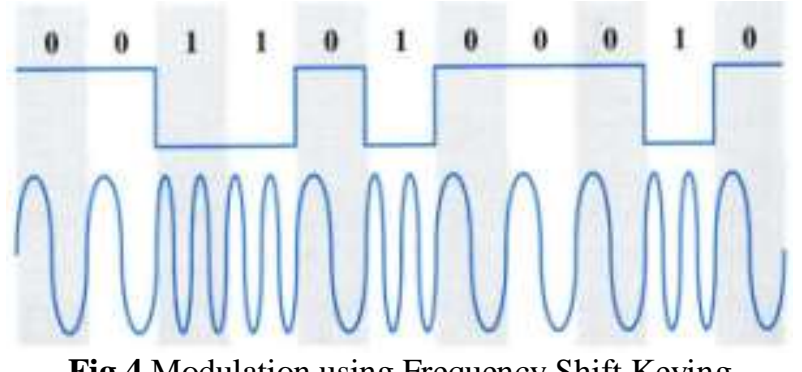

Fig 4 Modulation using Frequency Shift Keying

\section{APPLICATIONS}

\subsection{Military Application}

In military bases where particular base wants to connect the other or all the bases depending to the condition.

And during the war where all the bases needs to get connected to each other there this concept will be applicable.

\subsection{Educational Institutes}

When the particular message needs to be circulated to a particular class or every class the system will work appropriately and labor work will be saved. And most importantly the class will not get disturbed.

\subsection{Railways}

All the signal room and panel room they all will be having a individual receiver and their transmitter will be at master room so in case of emergency all will get connected. And all station will also get connected to each other.

\subsection{Conference Hall}

In conference hall also this idea of Wireless Audio Transmission and Switching System for Public Address is very useful. there the speakers mic will be connected to the multiple output speakers and it can cover a maximum area to deliver a talk. 


\subsection{Hospitals}

When doctor needs to get connected to the patient room or any room of the hospital, he can by using the Wireless Audio Transmission and Switching System for Public Address.

\section{ADVANTAGES}

\subsection{High Range}

The product concentrates on high range nearly 60-70 meters (LOS).

\subsection{Less Noise}

Due to low interference the noise will be minimum.

\subsection{Cost Friendly}

The components which we are using are simple and less costly.

\subsection{Low Maintenance}

Maintenance will be minimum.

\section{SCOPE}

1. The range of the system can be made for a very long distance communication if we transform the prototype into product.

2. The advantage of the system overtook the problem complexity as it covers the wider area and can do the efficient communication ove a very long distance than the conventional systems.

\section{CONCLUSION}

By overcoming the drawbacks of the previous existing technology we are going to make the better audio and switching system using RF modules and CC2500.

It will be of high range, cost efficient, compact in size and its maintenance will be easy.

Wireless Communication Technology is not useless, but it has certain limits. Wireless Communication may not be able to replace conventional radios completely, but it could enhance and charge the development of wireless communication and make it easier to spread a wireless signal across an house completely. At present, finding the exact position for a wireless router is difficult task. If the signal could be passed through VLC from Point A towards Point B inside a home, small local routers at both points could create local fields with less chance of overlapping and interfering with each other. Wide areas that are saturated with radio signals or that doesn't allow them for security reasons could use Wireless Communication as an alternate high-speed wireless network solution.

\section{REFERENCES}

[1]. https://en.wikipedia.org/wiki/RF_module

[2]. https://en.wikipedia.org/wiki/Wireless

[3]. https://en.wikipedia.org/wiki/Atmel_AT89_series

[4]. Design of transmitter and receiver.(Gutiérrez et al. EURASIP Journal on Advances in Signal Processing 2014, 2014:103http://asp.eurasipjournals.com/content/2014/1/103) [5]. http://www.tutorvista.com/content/physics/physicsiv/communication- systems/wireless-communication.php [6]. https://en.wikipedia.org/wiki/Project_Loon

[7]. http://www.dhgate.com/product/new-product-wtg-500uhf-wireless-acoustic/210885408.html

[8]. http://waytwolive.blogspot.in/2014/08/make-your-ownwireless-remote.html

[9]. https://en.wikipedia.org/wiki/ZigBee

[10]. http://www.amazon.in/Electret-Microphone-Inserts6050-Condenser/dp/B00NSP7G74?tag=googinhydr1841821 